\title{
On tracking the motion of rigid bodies through edge detection and least-squares fitting
}

\author{
Stuart J. Laurence
}

the date of receipt and acceptance should be inserted later

\begin{abstract}
A class of techniques is investigated for determining at least three components (two translational and one rotational) of the motion of a rigid body from silhouette images, with particular emphasis on motion within a fluid. The investigated techniques all employ edge detection followed by some form of least-squares fitting to the detected points in determining the movement of the body. Four techniques are discussed and, through both an artificial image analysis and calibrated sphere measurements, are shown to be capable of measuring displacements down to a few thousandths of a pixel under low image-noise conditions $(\lesssim 2 \%)$. Measurements of two configurations in a high-enthalpy shock tunnel demonstrate the capabilities of the techniques under experimental conditions. In particular, a technique referred to as edge-tracking is introduced, which can be employed in situations where the model profile is unknown and/or only some fraction of it is visible. This latter quality is especially useful for measurements in high-enthalpy facilities, where test-gas luminosity can obscure a significant extent of the model outline. A further advantage of this technique is that, even for complex geometries, the fitting procedure can typically be reduced to solving a sequence of linear least-squares problems, rather than a nonlinear one, with a corresponding benefit in computational efficiency.
\end{abstract}

S. J. Laurence

Spacecraft Department, Institute of Aerodynamics and Flow Technology, German Aerospace Center, Bunsenstr. 10, 37073

Göttingen, Germany

E-mail: stuart.laurence@dlr.de

\section{Introduction}

Imaging techniques are an attractive option for studying a variety of fluid flow problems, as they are inherently non-intrusive. The use of optical imaging to track the boundaries of non-rigid bodies such as bubbles and droplets is well established (Thoroddsen et al, 2008); imaging techniques have also been employed for the tracking of rigid bodies moving within a fluid, in applications as diverse as calculating the velocities of particles colliding with one another or with walls (Yang and Hunt, 2006; Marston et al, 2010), and measuring the forces on free-flying models in a shock tunnel (Warren et al, 1961; Bernstein, 1975). In such problems, the requirements are often somewhat different from those of typical computer-vision or medical-imaging applications for which many of the relevant image-processing techniques have been developed: the body geometries are usually well-defined, if not known a priori, and the emphasis is on precise displacement measurements so that velocities or accelerations can be derived with minimal uncertainty. While earlier image-based measurements using photographic film were limited in accuracy (Canning et al, 1970), recent work has shown that, with appropriate image-processing techniques applied to digital images, the motion of wind-tunnel-scale bodies can be tracked to the micron level (Laurence and Hornung, 2009; Laurence and Karl, 2010). Such accuracy allows precise time-resolved velocity and acceleration measurements despite the requisite differentiation of the signals.

Here I investigate the capabilities of a subset of image-based methods for the precise determination of a rigid body's motion, namely methods involving the detection of edge points on the body outline followed by some form of least-squares fitting to these. Such an 
approach calls for the visualization of the body silhouette, for which a backlighting arrangement or a technique such as shadowgraph or schlieren is appropriate. For measurements in compressible-flow wind tunnels, the ubiquity of shadowgraph/schlieren setups in these facilities is an advantage of the present approach over methods that require some other form of visualization, such as the tracking of fixed markers (Spoor and Veldpaus, 1980; Mettler, 2010). However, if only a single imaging device and optical path are employed, the number of components of the motion that can be measured is restricted, typically to two translational dimensions and about one rotational axis, though the extension to a second rotational component is discussed in this article. The unmeasured components of motion must then be assumed to be negligible, or, at least, to have a negligible influence on the image profile. Three-component measurements are still adequate for a large number of problems, however, and the addition of a second imaging device would allow the extension to further components.

Even assuming the use of silhouette images, the techniques under consideration here form only a subset of those available for deducing the motion of the visualized body. For example, phase-correlation methods employing the Fast Fourier Transform, commonly used in image registration, also provide such information (de Castro and Morandi, 1987). These methods consider entire regions of interest in an image, rather than making use of extracted features. However, the information in a silhouette image is restricted to the model edges, and edge-detection routines can be designed to account for the manner in which the edgepixels are created, an option not available for phasecorrelation techniques. It is thus difficult to see these latter techniques attaining the same levels of accuracy possible through those based on edge detection. Methods other than least-squares fitting are also available for determining the position of a body with known characteristics from a collection of edge points: one common alternative is the Hough transform (Ballard, 1981). However, least-squares techniques are more widely applicable, and, in the absence of significant outliers, are generally considered the best option (see, e.g., Zhang (1997)).

The edge-detection routine used throughout this work is described in Laurence and Karl (2010): following an initial pixel-resolution Canny detection (Canny, 1986), a subpixel detection is performed that was shown to allow the location of straight edges in a noise-free image to $\sim 1 / 50$ th of a pixel. The most serious limitation of this implementation is the systematic error that results when the Gaussian smoothing step is applied to highly curved edges (Verbeek and van Vliet, 1994). In the present article, this subpixel detector is used in conjunction with four least-squares techniques to determine the motion of a rigid body in both artificially constructed and experimentally obtained images. The accuracies of the techniques are analyzed, and their relative merits and deficiencies discussed.

\section{Descriptions of tracking techniques}

In this section the four least-square techniques considered in this work are described. To begin, we limit ourselves to motions consisting only of a rotation and a linear translation within the imaging plane, and assume there to be no distortion present in the imaging system. Out-of-plane rotations and other transformations resulting in profile distortions are discussed in the following section.

\subsection{Analytic-fitting}

If the visualized cross-sectional profile is known and can be expressed as a single-valued analytic function, $r(\theta)$, the body-fitting technique first introduced in Laurence and Hornung (2009) can be used. This technique may be considered a generalization of the nonlinear methods for circles and ellipses described in Gander et al (1994). For the locus of detected edge points in each image, values of the center-of-geometry position, the rotation angle, and the scaling factor resulting in the minimum error with the known $r(\theta)$ (in the least-squares sense) are determined by an iterative fitting procedure. Highly accurate position measurements have been shown to be possible with this technique (Laurence and Karl, 2010), and it will be considered as the baseline for comparison to the other techniques discussed herein.

\subsection{Spline-fitting}

It is not always the case that the visualized profile is such that the analytic-fitting technique can be employed: for example, the profile might be defined by a collection of points in a CAD model rather than by an analytic curve, significant optical distortions might be present, or the precise shape might simply be unknown. In such cases, the profile can instead be defined by a set of points, $r_{i}(\theta)$, obtained either from the CAD model or from a reference image in the obtained sequence; then, during the iteration process, splines or some other form of interpolation can be used to provide a continuous profile between the $r_{i}$. As in the analytic-fitting case, a 
careful choice of the center-of-geometry may be necessary to ensure this $r$ is a single-valued function of $\theta$.

\subsection{Edge-tracking}

The two techniques described thus far share a major disadvantage: in general, they both involve a nonlinear least-squares problem, and thus call for a timeconsuming iterative solution procedure. Also, in practice, the fitting procedure in each case typically requires that the edge points cover a substantial fraction of the body profile in order to converge reliably to a solution; this is particularly the case if the scaling factor between image and physical dimensions is to be determined as part of the fit. This latter requirement can be problematic, for example, in measurements on blunt bodies in high-enthalpy shock tunnels, where test-gas luminosity can obscure the entire front face of the model (see figure 11).

To overcome these two difficulties, a new technique has been developed which tracks only the motion of visible edges, rather than attempting to fit the entire body profile. The movement between images, rather than an absolute position in image space, is thus obtained. This edge-tracking technique is based on first matching closest edge points in the two images of interest, which are referred to hereinafter as the reference and displaced images, and then determining the range of possible movements corresponding to the relative point positions and the edge angles. A least-squares fit is then carried out over the movement space to determine the most likely values.

To illustrate the edge-tracking technique, we first concentrate on motions in which either the visualized profile is rotationally symmetric (i.e. circular) or rotation of the profile between images is negligible. Consider the situation shown in figure 1: a body defined by three edge points (black), each with an edge angle $\varphi$ (assumed known), undergoes a horizontal motion to the right of magnitude 0.5 pixels. As subpixel detection routines typically resolve the edge position in the direction normal to the edge, the displaced edge points (gray) correspond to different positions on the body outline. However, if the edge angle between each reference $\left(x_{e}, y_{e}\right)$ and displaced $\left(x_{e}^{\prime}, y_{e}^{\prime}\right)$ point-pair is assumed to remain unchanged, the range of possible translations $(\Delta x, \Delta y)$ for each pair must satisfy the equation

$\Delta x \sin \varphi-\Delta y \cos \varphi=\left(x_{e}^{\prime}-x_{e}\right) \sin \varphi-\left(y_{e}^{\prime}-y_{e}\right) \cos \varphi$.

The curves in movement space corresponding to the possible motions for the three point-pairs shown are presented in the left lower plot of figure 1; as expected, they intersect at the correct values: $\Delta x=0.5, \Delta y=0$. For real images, however, there is invariably some error associated with determining the edge-point positions and edge angles; in this case, a solution can be obtained by solving the linear least-squares problem given by (1) in which $\varphi, x_{e}, y_{e}, x_{e}^{\prime}$, and $y_{e}^{\prime}$ are all vectors. A solution in which errors have been added to the point-pair displacements is shown in the right lower plot of figure 1.
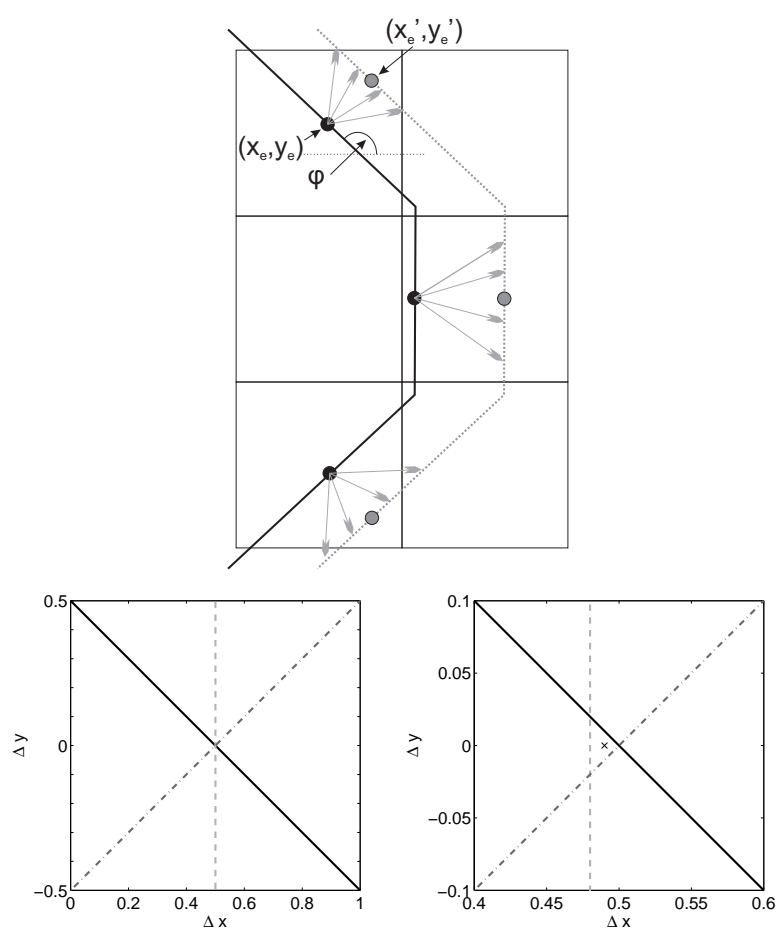

Fig. 1 Edge-tracking without rotation: each reference edge point (black) has a range of possible motions defined by its nearest neighbor in the displaced image (gray) and its edge angle. The possible range of displacements for the three edge-point pairs are shown in the left lower plot; in the absence of any errors, the solution is the intersection of the three lines. The least-squares solution with errors present is shown to the right.

A more general edge-tracking description can be formulated to cover motions in which, in addition to a translational displacement, transformations such as rotations are present. If the transformation in question maps the point $\left(x_{e}, y_{e}\right)$ onto $\left(x_{i}, y_{i}\right)$, and the edge angle $\varphi$ onto $\varphi^{\prime}$, a generalized edge-tracking equation may be written

$\left(x_{i}+\Delta x-x_{e}^{\prime}\right) \sin \varphi^{\prime}=\left(y_{i}+\Delta y-y_{e}^{\prime}\right) \cos \varphi^{\prime}$,

where the other notation is as before. The major assumption here is that the visualized edges are locally straight, so that $\varphi^{\prime}$ is the edge angle of both $\left(x_{i}, y_{i}\right)$ and $\left(x_{e}^{\prime}, y_{e}^{\prime}\right)$, though they will not in general correspond to 
the same physical location on the edge. Thus, significant errors are likely to be incurred at points on edges with high degrees of curvature and, in general, it is recommended that such points be excluded from the least-squares fit.

Specializing now to the case of rotations within the imaging plane, consider figure 2: a reference edge point $\left(x_{e}, y_{e}\right)$ with a known edge angle $\varphi$ is assumed to undergo a rotation $\alpha$ about a known center-of-rotation, assumed here to lie at the origin (in any case, the edge points can be shifted so that this holds), and then a translation of $(\Delta x, \Delta y)$. Thus, $\varphi^{\prime}=\varphi+\alpha, x_{i}=x_{e} \cos \alpha-$ $y_{e} \sin \alpha$, and $y_{i}=x_{e} \sin \alpha+y_{e} \cos \alpha$. The possible values of $\Delta x, \Delta y$, and $\alpha$ are then determined by the position of the closest edge point in the displaced image, $\left(x_{e}^{\prime}, y_{e}^{\prime}\right)$, and the edge-tracking equation, (2), becomes

$$
\begin{aligned}
& \Delta x \sin (\varphi+\alpha)-\Delta y \cos (\varphi+\alpha)= \\
& \sin (\varphi+\alpha)\left[x_{e}^{\prime}-x_{e} \cos \alpha+y_{e} \sin \alpha\right]- \\
& \cos (\varphi+\alpha)\left[y_{e}^{\prime}-y_{e} \cos \alpha-x_{e} \sin \alpha\right] .
\end{aligned}
$$

An alternative form of (3) is obtained by replacing $\alpha$ with $\varphi^{\prime}-\varphi$.

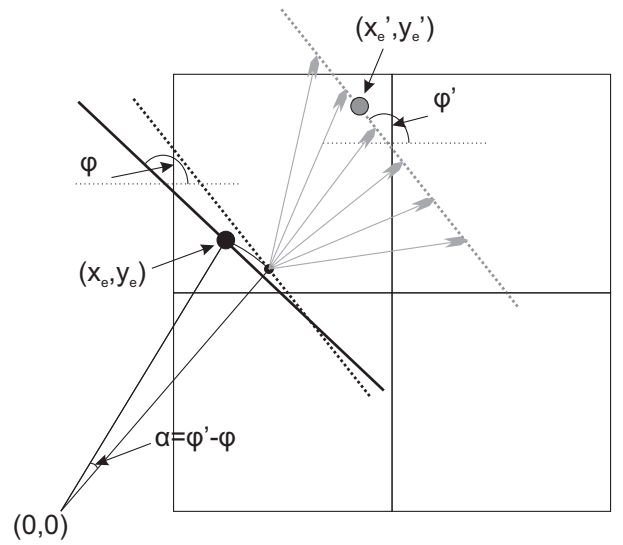

Fig. 2 Edge-tracking with an in-plane rotation: an edge point $\left(x_{e}, y_{e}\right)$ is assumed to undergo a rotation $\alpha$ about a given centerof-rotation (assumed here to lie at the origin), and then a translation to a point on the line determined by the point $\left(x_{e}^{\prime}, y_{e}^{\prime}\right)$ and the direction $\varphi^{\prime}=\varphi+\alpha$.

With $x_{e}, y_{e}, x_{e}^{\prime}, y_{e}^{\prime}, \varphi$ (and possibly $\varphi^{\prime}$ ) as vectors corresponding to point-pair locations and directions in the reference and displaced images, (3) takes the form of a least-squares problem. If $\varphi$ and $\varphi^{\prime}$ are both known, the alternative form of (3) can be used, and the problem reduces to a linear one for $\Delta x$ and $\Delta y$. In practice, however, using both $\varphi$ and $\varphi^{\prime}$ was not found to be satisfactory: much-improved results were obtained if only one of $\varphi$ or $\varphi^{\prime}$ were specified and $\alpha$ determined by other means. This may be due to the fact that the edge angles, since they are derived from differences in edge locations, cannot be determined as accurately as the edge locations themselves. One obvious option is to solve the full nonlinear problem (3) iteratively; however, this can be time-consuming and it is desirable to employ other means to reduce (3) to a linear problem. Two such approaches are proposed here.

The first approach is possible for any body which has a straight edge section on some part of its visualized profile; in this case, it is possible to determine $\alpha$ independently from $\Delta x$ and $\Delta y$ by fitting a straight line to this section in each of the reference and displaced images. With $\alpha$ known, (3) then reduces to a linear problem in $\Delta x$ and $\Delta y$; alternatively, if the reference edge points are rotated by $\alpha$ prior to edge-point matching, (1) can be used in place of (3). In practice, the latter option was found to be slightly more effective, perhaps because of reduced numerical error. While this approach to linearizing the problem, hereinafter referred to as straight-edge linearization, is not possible for all model geometries, it was found to be effective in the experiments described in this work.

The second approach, possible for arbitrary geometries, is to linearize (3) by assuming the rotation angle and the displacements to be small. First, assuming $\alpha \ll 1$, we may approximate $\cos \alpha \approx 1$ and neglect terms in $\sin ^{2} \alpha$. Equation (3) then becomes

$$
\begin{array}{r}
\Delta x(\sin \varphi+\cos \varphi \sin \alpha)- \\
\Delta y(\cos \varphi-\sin \varphi \sin \alpha)-\sin \alpha\left(x_{e}^{\prime} \cos \varphi+y_{e}^{\prime} \sin \varphi\right)= \\
\left(x_{e}^{\prime}-x_{e}\right) \sin \varphi-\left(y_{e}^{\prime}-y_{e}\right) \cos \varphi \cdot(4)
\end{array}
$$

To reduce (4) to a form to which a linear fit can be applied, we must make the further assumption that $\Delta x$ and $\Delta y$ are small in comparison to $x_{e}^{\prime}$ and $y_{e}^{\prime}$, respectively. With this assumption, a linear least-squares problem for $\Delta x, \Delta y$, and $\sin \alpha$ is obtained:

$$
\begin{array}{r}
\Delta x \sin \varphi-\Delta y \cos \varphi-\sin \alpha\left(x_{e}^{\prime} \cos \varphi+y_{e}^{\prime} \sin \varphi\right)= \\
\left(x_{e}^{\prime}-x_{e}\right) \sin \varphi-\left(y_{e}^{\prime}-y_{e}\right) \cos \varphi .
\end{array}
$$

In section 4.1, the accuracy of this linearized equation is analyzed with artificial images. Its ability to determine $\Delta x$ and $\Delta y$ is found to be limited, but, for translational displacements up to $\sim 5$ pixels, its accuracy in determining small $\alpha$ is very good. Thus, a recommended method for solving the nonlinear equation (3) is to first solve (5) for $\alpha$, then, as in the previous (straight-edge) approach, to use this value to determine $\Delta x$ and $\Delta y$ either from (3) or from (1) following a rotation of the reference edge points. In high-speed image sequences, displacements and rotations between consecutive images will often be small enough that this linearized approach can be employed. 


\subsection{Corner-tracking}

The fourth technique is suitable for a limited class of bodies, namely those having at least two non-parallel straight-edge sections on the visualized cross-section. Even if these edges do not intersect physically, the lines describing them intersect at a virtual corner, and the motion of this, together with the change in angle determined from the edge sections themselves, can be used to infer the motion of the body as a whole. Least-squares fitting is an obvious candidate for determining the equations of the lines corresponding to the edges. The accuracy of this technique will depend on the geometry of the body: in particular, the extent of the straight-edge sections, the angle between them, and the distance from the (virtual) corner to the edge sections.

\section{Out-of-plane rotations and other transformations}

\subsection{Out-of-plane rotations}

Dealing with rotations out of the imaging plane is somewhat more complicated than in-plane rotations, and since the resulting change in the visualized silhouette will be strongly dependent on the three-dimensional profile of the body, it is impossible to give a general prescription. The present techniques will typically not be as accurate in determining a small out-of-plane rotation, $\gamma$, as a comparable in-plane rotation, $\alpha$, as the shift in the edge-point location due to the former will be smaller. For edges lying close to the imaging plane, for example, the edge-point shift will scale roughly as $1-\cos \gamma \approx \gamma^{2}$ for in-plane and $\sin \alpha \approx \alpha$ for out-of-plane rotations. By the same token, however, uncertainty in the out-of-plane rotational angle will not be as detrimental to the accurate determination of translational displacements, if these are the quantities of main interest.

For simple geometries (e.g., cylinders, cones, and flat bodies) the extension of the analytic-fitting technique to out-of-plane rotations is straightforward. However, for more realistic geometries (even those for which the cross-section can be easily expressed analytically, such as the capsule geometry considered in section 4.1.2), deriving a full three-dimensional analytic description may be impractical or impossible. In this case, some form of three-dimensional spline-fitting technique might be possible, but is likely to be significantly more computationally expensive.

Employing the edge-tracking technique for out-ofplane rotations is slightly less restrictive than analyticfitting, as it is only changes to the edge-point loca- tions due to the rotation that must be described analytically, rather than the locations themselves (a flat body of otherwise unknown shape is one example in which edge-tracking but not analytic-fitting could be utilized). Note, however, that because the edge-point location varies as approximately $\gamma^{2}$ rather than $\gamma$, the initial angle must be known to determine the subsequent rotation angle, in contrast to in-plane rotations. For even moderately complex body geometries, it is unlikely that the requisite analytic description is possible, and it will be more important to estimate the effect that an unknown out-of-plane rotation will have on the measured displacements.

For certain simple geometries, however, an edgetracking equation can be formulated to describe out-ofplane rotations. As one example, consider the case of a flat body (or quasi-flat body with sharp edges), initially aligned with the imaging plane, undergoing a rotation $\gamma$ about either the $x$ - or $y$-axis. For rotation about the $y$-axis, $x_{i}=x_{e} \cos \gamma$ and $\tan \varphi^{\prime}=\tan \varphi / \cos \gamma$; (2) becomes

$$
\begin{array}{r}
\Delta x \sin \varphi-\Delta y \cos \gamma \cos \varphi+ \\
\cos \gamma\left[x_{e} \sin \varphi+\left(y_{e}^{\prime}-y_{e}\right) \cos \varphi\right]=x_{e}^{\prime} \sin \varphi .
\end{array}
$$

Treating $\Delta y \cos \gamma$ as one of the least-squares variables, this equation can be solved immediately. A similar equation may be derived for rotations about the $x$-axis.

If the rotation is about an axis lying at an angle $\psi$ (assumed known) to the $y$-axis in the $x$ - $y$ plane, however, the equations become more complicated. We then have $\tan \left(\varphi^{\prime}-\psi\right)=\tan (\varphi-\psi) / \cos \gamma$, and

$x_{i}=x_{e}-(1-\cos \gamma) \cos \psi\left(x_{e} \cos \psi+y_{e} \sin \psi\right)$, $y_{i}=y_{e}-(1-\cos \gamma) \sin \psi\left(x_{e} \cos \psi+y_{e} \sin \psi\right)$.

Substituting into (2), we obtain the following nonlinear equation:

$$
\begin{array}{r}
\mathcal{N}_{1} \Delta x-\mathcal{N}_{2} \Delta y=\mathcal{N}_{1}\left(x_{e}^{\prime}-x_{e}\right)-\mathcal{N}_{2}\left(y_{e}^{\prime}-y_{e}\right)+ \\
(1-\cos \gamma)\left(x_{e} \cos \psi+y_{e} \cos \psi\right) \sin (\varphi-\psi),
\end{array}
$$

where

$\mathcal{N}_{1}=\sin \varphi-(1-\cos \gamma) \sin \psi \cos (\varphi-\psi)$,

$\mathcal{N}_{2}=\cos \varphi-(1-\cos \gamma) \cos \psi \cos (\varphi-\psi)$.

The linearized form of this equation, obtained by neglecting terms of the order $(1-\cos \gamma)^{2}$ and assuming $\Delta x$ and $\Delta y$ to be small, is

$$
\begin{array}{r}
\Delta x \sin \varphi-\Delta y \cos \varphi+\cos \gamma\left[x_{e} \sin \varphi-\right. \\
\left.y_{e} \cos \varphi-\left(x_{e}^{\prime} \sin \psi-y_{e}^{\prime} \cos \psi\right) \cos (\varphi-\psi)\right]= \\
\left(x_{e}^{\prime} \cos \psi+y_{e}^{\prime} \sin \psi\right) \sin (\varphi-\psi) .
\end{array}
$$

In contrast to the corresponding equation for inplane rotations, (8) is found to give a good approximation to the rotational angle only for small $\Delta x$ and 
$\Delta y$. Thus, the recommended approach here is to solve the non-rotational edge-tracking equation, (1), for $\Delta x$ and $\Delta y$, shift the reference edge points by this amount, and then apply the linearized equation (8) to determine the rotational angle. Subsequently, $\Delta x$ and $\Delta y$ can be determined more accurately with the full equation (7).

For flat bodies, it is also possible to construct an edge-tracking equation describing a combination of inplane and out-of-plane rotations. This equation can be linearized, allowing the determination of the rotational angles, and as before, these can be used to solve the full equation for the linear displacements.

\subsection{Optical distortions and non-rotational transformations}

Thus far it has been implicitly assumed that the silhouette images have been created by parallel light, such as in a schlieren or shadowgraph setup, and thus that no perspective effects (i.e., parallax) are introduced. Optical distortions such as astigmatic aberration are invariably present in such systems (see, e.g., Settles (2006)), but these can be corrected for relatively easily, for example, by visualizing an orthogonal grid in the imaging plane. However, for visualization methods such as backlighting, unless a specialized telecentric lens is employed, perspective errors that are not so easily corrected for will be present.

The effects of parallax are similar in some ways to those of out-of-plane rotations, and, for very simple geometries, it may be possible to account for them in a similar manner; this is especially true of the edgetracking technique, with its less stringent demands with regard to the required knowledge of the body geometry. As one example, consider a cube of side length $2 l$ placed at a distance $d$ from the camera with its edges aligned with the image axes. Concentrating first on the cube's vertical edges, as shown in figure 3 , if the physical locations of the inner and outer edges (relative to the centerline of the imaging device) are $x_{1}$ and $x_{2}$, the visualized locations will be $\hat{x}_{1}=x_{1} /(1+l / d)$ and $\hat{x}_{2}=x_{2} /(1-l / d)$, respectively, i.e., the edge positions undergo a linear scaling in the $x$-direction. The horizontal edges will undergo an identical scaling in $y$; the visualized non-orthogonal segments connected to any inner edges will in general be short and can be excluded from the fit. An edge-tracking equation for this case can then be written as

$$
\begin{array}{r}
\Delta x \sin \varphi-\Delta y \cos \varphi \pm l / d \times\left(\hat{x}_{e}^{\prime}-\hat{x}_{e}\right) \sin \varphi= \\
\pm l / d \times\left(\hat{y}_{e}^{\prime}-\hat{y}_{e}\right) \cos \varphi+\left(\hat{x}_{e}^{\prime}-\hat{x}_{e}\right) \sin \varphi- \\
\left(\hat{y}_{e}^{\prime}-\hat{y}_{e}\right) \cos \varphi
\end{array}
$$

The plus and minus signs here correspond to outer and inner edges, respectively; the term immediately to the left of the equals sign applies only to vertical edge points, that to the right only to horizontal edge points. But, since all considered edges are either vertical or horizontal, these may be considered separately, and (9) can be reduced to a pair of equations, one for each of $\Delta x$ and $\Delta y$, i.e.,

$$
\begin{aligned}
\Delta x \pm l / d \times\left(\hat{x}_{e}^{\prime}-\hat{x}_{e}\right) & =\hat{x}_{e}^{\prime}-\hat{x}_{e} \\
\Delta y \pm l / d \times\left(\hat{y}_{e}^{\prime}-\hat{y}_{e}\right) & =\hat{y}_{e}^{\prime}-\hat{y}_{e} .
\end{aligned}
$$

If $l / d$ is known, it can be substituted into these equations; if not, it can be included as one of the variables in the least-squares fit. In the latter case, however, the scaling will be indistinguishable from an out-of-plane rotation.

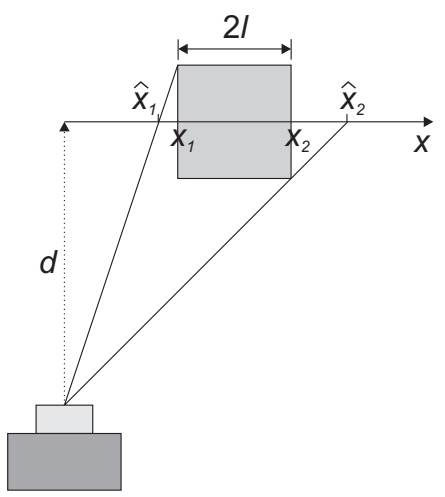

Fig. 3 Illustration of the parallax errors introduced in tracking an aligned cube with a conventional visualization setup.

This is a somewhat trivial example, and indeed, the relevant equations could be formulated without going through the edge-matching methodology, but it illustrates how the the edge-tracking technique can be employed for image transformations other than translations and rotations; a shear transformation could be included in a similar manner. However, the types of geometries that can be so accounted for are limited, and, as with out-of-plane rotations, in practice it is likely to be more important to estimate the errors that optical distortions will produce in the tracking techniques if they are applied in an unmodified form. An example of such an estimation is given in section 4.1.3. To avoid these errors, the use of a telecentric lens is recommended where possible. 


\section{Estimation of tracking accuracy}

\subsection{Artificial images}

Artificial images have been shown to be a useful tool for estimating the errors in image-based tracking techniques (Laurence and Karl, 2010). In this subsection such images are employed to estimate the errors in the four tracking techniques under both noise-free and noisy conditions, considering configurations both in which rotations are unimportant (sphere images) and in which they play an important role.

The construction of artificial images here is as described in Laurence and Karl (2010). Briefly, high and low intensity values are first chosen, corresponding to pixels completely outside and completely inside the body silhouette. For the specified body size and position, all intersection points of the body's outline with pixel boundaries are calculated; the intensities of the edge pixels are then determined by the weighted averages of the pixel areas outside and inside the dividing curve (defined by the intersection points on the pixel boundary) with the high and low intensity values. All other intensities are specified as either the high or the low value, and Gaussian noise is added if desired.

\subsubsection{Sphere images}

First, artificial sphere images are utilized to determine the errors in the non-rotational versions of the various techniques. The two techniques that employ a fitted profile to the image edge points have errors that are (within the pixellation error) independent of the displacement of the object. For edge-tracking, however, this is not the case, as the assumption that matched edge points in the two images share the same physical edge angle becomes more and more doubtful as the displacement is increased, particularly along edges with high degrees of curvature. Thus, errors will grow as the object displacement between reference and displaced images is increased. However, this source of error can be addressed by repeating the edge-point matching with the reference edge points shifted by the displacements calculated from the first least-squares fit, and then repeating the fit. For large displacements between images, this correction might have to be performed repeatedly, especially for complex body profiles.

To quantify the errors both with and without this suggested correction, edge-tracking was applied to noisefree artificial sphere images. The $y$-location of the sphere center was kept constant while the $x$-location was varied over a range of 5 pixels; this was repeated for 50 $y$-values, evenly spaced over a range of 0.5 pixels (the images being symmetric every half-pixel), and the mean error in the total displacement, $\sqrt{\delta x^{2}+\delta y^{2}}$, at each $x$-location was calculated. The results for sphere radii of 20 and 100 pixels, both for corrected and uncorrected edge-tracking (i.e, with and without repeated edge-point matching) are shown in figure 4, together with those for analytic-fitting (note that a linear version of this technique is available for circular/spherical geometries). For both sphere radii the uncorrected error is seen to rise rapidly from between 0.5 and 1 pixel. The error for the smaller radius reaches a value of 0.1 pixels or $2 \%$ after 5 pixels; increasing the radius to 100 pixels decreases this maximum error by roughly an order of magnitude. The suggested correction successfully addresses the source of this error, however. The error for analytic-fitting also remains roughly independent of the displacement, as expected. For the smaller radius, the errors for analytic-fitting and corrected edgetracking are very close, but analytic-fitting exhibits notably smaller errors for the larger radius.

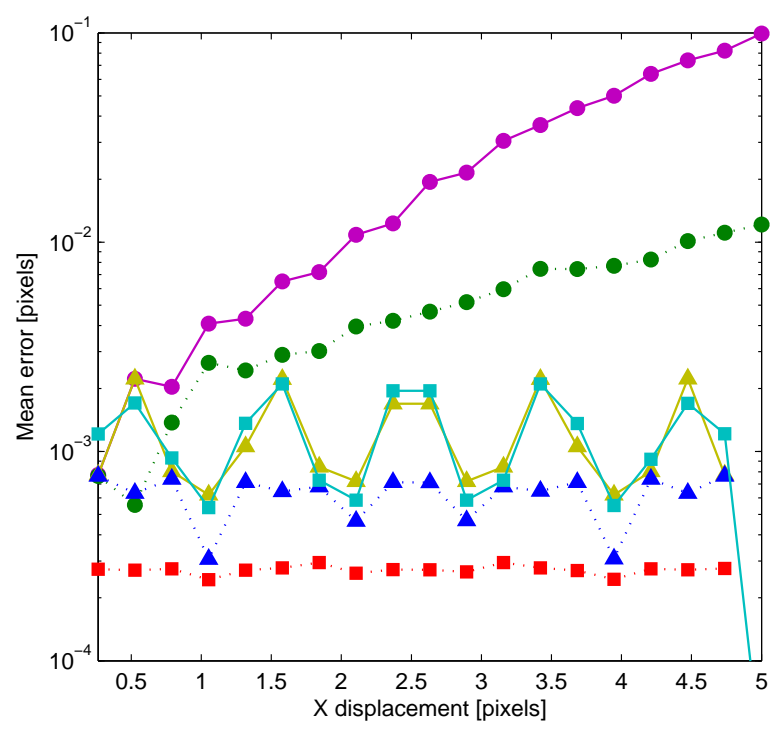

Fig. 4 Errors in displacement measurements for noise-free artificial sphere images using the uncorrected and corrected edgetracking techniques ( $\mathrm{a}$ and $\triangle$, respectively), and analytic fitting $(\square)$. The mean error is the combined $x$ and $y$ error, averaged over 50 different $y$-values. The sphere radii are 20 (solid) and 100 (dotted) pixels.

All real images contain some degree of noise, which adversely affects the accuracy of any image-based technique. In figure 5 are plotted the mean errors in the three applicable techniques as functions of the sphereimage noise level. At each noise value, the average error in displacement measurements between a reference image and 400 displaced images - with the sphere cen- 
ter varied over all positions on a regularly distributed $20 \times 20$ grid of dimensions $1 \times 1$ pixel - is shown. Three sphere radii of 20, 50, and 100 pixels are compared. For low noise levels, analytic-fitting consistently exhibits the smallest error (there is no consistent trend favoring either spline-fitting or edge-tracking over the other), but gradually converges to the other techniques as the noise is increased. This convergence occurs later for larger values of the sphere radius, but for noise levels above $\sim 1 \%$ the three techniques are all but indistinguishable. These results indicate that, provided the noise level is less than $\sim 2 \%$, measurements to within a few thousandths of a pixel are attainable with any of these techniques.

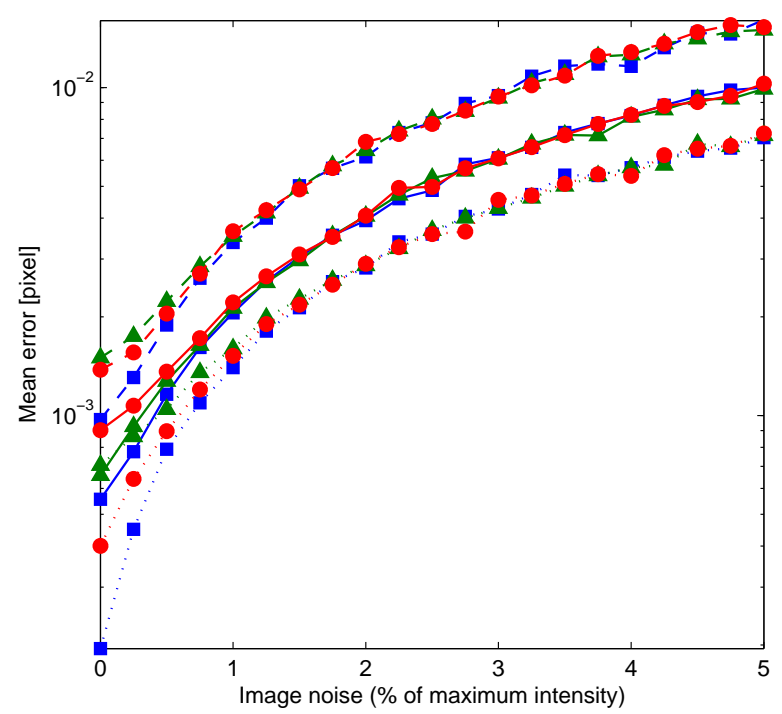

Fig. 5 Effect of image noise on errors for the three relevant techniques applied to artificial sphere images: analytic-fitting ( $\square$ ), spline-fitting $(\triangle)$, and corrected edge-tracking $(\circ)$. The sphere radii are 20 (dashed), 50 (solid), and 100 (dotted) pixels.

\subsubsection{Capsule images}

Artificial images of the capsule model shown in figure 11 (minus the sting attachment) were used to characterize the accuracy of the various techniques when in-plane rotational motions are present.

As for translational errors in the rotation-free case, analytic- and spline-fitting will have rotational errors that are independent of the degree of rotation. For uncorrected edge-tracking using (3), however, increasing the rotational angle between images, and thus also the mean distance between reference and displaced edge points, will lead to the same problem that affected purely translational measurements. To show this, in figure 6 are plotted the total displacement errors, $\sqrt{\delta x^{2}+\delta y^{2}}$, for various tracking techniques applied to 200-pixel diameter noise-free capsule images as the rotation angle between the reference and displaced images is varied (the rotational origin here is the capsule center-ofgeometry). To produce these error curves, for each rotation angle the mean error was calculated over 400 displaced images, with the capsule center being varied over all positions on a $20 \times 20$ regular grid of $1 \times 1$ pixel dimensions (for analytic fitting, a $10 \times 10$ grid of dimensions $0.5 \times 0.5$ pixels is used). The errors for each technique, averaged over all rotation angles, are tabulated in table 1 for capsule diameters of 100, 200, and 400 pixels. Edge-tracking with straight-edge linearization using both the full model profile and only the rear edges (as in the measurements in section 5.1) was employed, both with and without the correction suggested in the previous section, i.e., repeated matching of the edge points. Analytic fitting was applied both to full profiles and to profiles with points on the capsule corners and shoulders removed, the latter to pinpoint the effect of edges with high degrees of curvature (for which the subpixel detection routine exhibits a systematic error) on the tracking accuracy. Corner-tracking was also applied to the rear edges.

The error for uncorrected edge-tracking is seen to rise monotonically with increasing rotation angle, reaching a value of approximately 0.02 pixels for a rotation of $1^{\circ}$. However, the use of repeated edge-point matching is seen to successfully address this problem, with the corresponding profile (corrected, full profile) exhibiting a roughly constant error as the rotation angle is increased. The use of this correction for all edgetracking variants is thus assumed hereinafter. A further improvement in accuracy is possible if only the rear edges are employed. The detrimental effect of including highly curved edges is especially pronounced for analytic-fitting, and suggests that edge-tracking might be more robust to systematic errors in the edge location in some cases. In contrast to the sphere images, it is apparent that analytic-fitting is no more accurate here than corrected edge-tracking. This may be attributed to the predominance of straight edges on the capsule geometry, for which edge-tracking would be expected to produce minimal error. In fact, if only the rear (straight) edges are used, table 1 shows that even uncorrected edge-tracking is as accurate as any of the technique variants. Errors with corner-tracking are seen to be generally higher than those of the other techniques: this is especially pronounced for smaller image dimensions.

To determine the effectiveness of the linearized edgetracking equation, (5), this was also applied to the cap- 


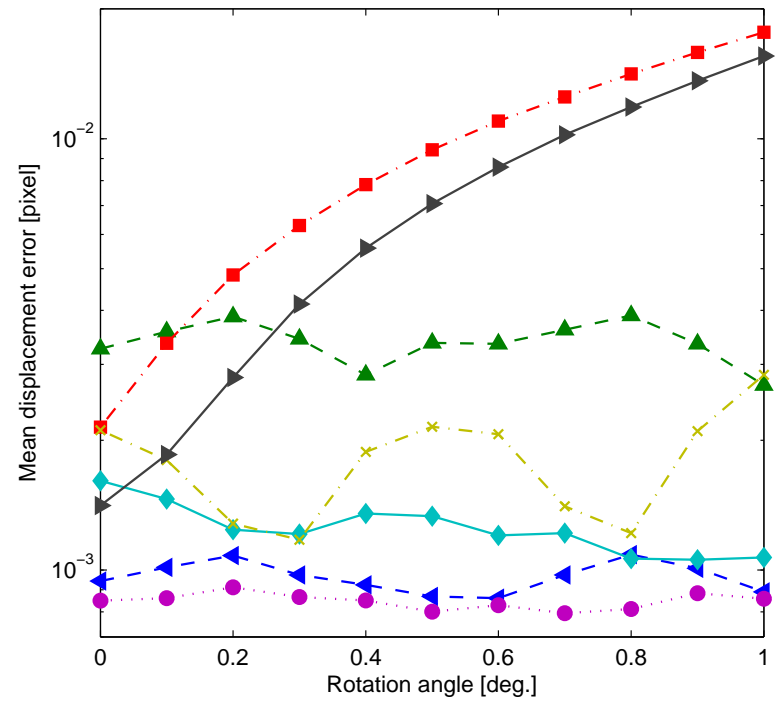

Fig. 6 Mean errors in the position determination of artificial noise-free capsule images of diameter 200 pixels as the rotational angle between images is varied: o, corrected edge-tracking with straight-edge linearization(rear edges only); $\diamond$, corrected edgetracking with straight-edge linearization (full profile); $\square$, uncorrected edge-tracking (full profile); $\triangleright$, linearized edge-tracking, no repeated fit (full profile); x, corner-tracking; $\triangleleft$, analytic-fitting (corners and shoulders excluded); $\triangle$, analytic-fitting (full profile).

\begin{tabular}{lccc} 
& \multicolumn{3}{c}{ Capsule diameter (pixels) } \\
Technique & 100 & 200 & 400 \\
\hline & & & \\
Corrected edge-tracking (rear) & 1.6 & 0.85 & 0.41 \\
Corrected edge-tracking (full) & 2.7 & 1.26 & 0.77 \\
Uncorrected edge-tracking (rear) & 1.5 & 0.77 & 0.40 \\
Uncorrected edge-tracking (full) & 11.1 & 9.5 & 7.5 \\
Corner-tracking & 6.9 & 1.8 & 0.89 \\
Analytic-fitting & 1.1 & 0.97 & 0.54 \\
(no corners/shoulders) & & & \\
Analytic-fitting (full) & 3.9 & 3.3 & 2.1
\end{tabular}

Table 1 Mean displacement errors (in thousandths of a pixel) for measurements on noise-free artificial capsule images of different sizes, using variants of the present techniques. The errors are averaged over measurements involving both varying translational and rotational motions, e.g., each of the profiles shown in figure 6 .

sule images. The result obtained using only the linearized equation (without a subsequent refit for $\Delta x$ and $\Delta y$ ) is also shown in figure 6 . It is clear that using only this equation to determine the displacements leads to large errors for anything more than small rotations; this error was found to become progressively worse as the maximum displacement increased. However, when the rotational angle determined from the linearized equation was subsequently substituted into the original equation to solve again for the displacements, as recommended in section 2.3 , the results were essen- tially identical to those obtained with straight-edge linearization. This was found to hold to displacements of at least 5 pixels, though increasing much beyond this point led to increased errors in the linearized result. However, since we are free to shift the edge points as we choose prior to carrying out the fitting operations, \pm 5 pixels should give a sufficiently large margin of error for this strategy to be effective at larger displacements also.

The accuracy in determining the rotation angle for noiseless images was found to be similar amongst the various techniques, each capable of resolving the angle to approximately $0.001^{\circ}$ for 200 -pixel diameter capsule images.

To explain why the linearized equation gives significantly better results for the rotational angle than for the translational displacements, note that the reduction from equation (4) to (5) is valid even for large displacements if both $\cos \varphi \sin \alpha \ll \sin \varphi$ and $\sin \varphi \sin \alpha \ll$ $\cos \varphi$. Even for small $\alpha$, however, these two conditions cannot hold together for $\varphi$ close to $0, \pm \pi / 2$, or $\pm \pi$. Concentrating first on points for which either $\varphi \approx 0$ or $\pm \pi$, we see that $\varphi \sin \alpha \ll \sin \varphi$ fails to hold, and as the relevant term multiplies $\Delta x$, this will lead to errors in the determination of $\Delta x$ for these points. However, in this case, (4) reduces to leading order to an equation in only $\sin \alpha$ and $\Delta y$, and the effect of this error on the determination of both $\sin \alpha$ and $\Delta y$ will be small. On the other hand, if $\varphi$ is instead close to either $\pm \pi / 2$, the roles of $\Delta x$ and $\Delta y$ are reversed; however, in either case, the error incurred for $\sin \alpha$ is small. Thus, provided $\alpha$ is small, the fully linearized problem (5) gives a good approximation for $\sin \alpha$, even for non-small $\Delta x$ and $\Delta y$.

An analysis of capsule images with noise yielded results for analytic-fitting and edge-tracking similar to those seen in figure 5. The corner-tracking technique was found to be particularly susceptible to noise, with the displacement error at a given noise level being typically twice as large as those of the other techniques. The error in the rotation angle for techniques that employ only straight edges to determine the angle (i.e., edgetracking with straight-edge linearization and cornertracking) was also found in general to be higher than for techniques that employed a larger fraction of the body profile. For example, at $2 \%$ noise, the mean error for linearized edge-tracking was approximately $0.0025^{\circ}$, compared to $0.0055^{\circ}$ for the straight-edge-based techniques. This may be attributed to the smaller number of points used to determine the angle for the latter techniques. However, the translational displacement errors were generally not affected by this increased rotational error. 


\subsubsection{Cone images}

Noise-free cone images were employed to characterize the errors in the present techniques when out-of-plane rotations are present. A conical geometry was chose because it is simple enough to allow an analytical description under such rotations, but the geometrical asymmetry means that errors from either end of the body will not cancel each other out, as would be the case for a circular cylinder, for example. For all measurements described here, a $20^{\circ}$ half-angle conical geometry with a base radius of 100 pixels was employed. In figure 7, the displacement errors recorded for several variants of the tracking techniques are plotted against the rotational angle (with zero angle corresponding to a side-on profile). The points here are not averaged but rather correspond to single measurements in which the cone vertex remained fixed; the center-of-rotation was assumed to lie $1 / 4$ of the cone length from the base, appropriate for a solid cone of uniform density.

First, comparing the two edge-tracking variants one in which the cone base edge points are included, and the other in which these points are excluded - inclusion of the base points is seen to lead to a rapidly growing error as the rotation angle is increased, but this error is dramatically decreased if the base is excluded. In the latter case, the displacement error remains below two-thousandths of a pixel for rotations up to $0.5^{\circ}$, whereas the error for included base points has grown to 0.5 pixels for this rotation angle. Results from analyticfitting both with and without out-of-plane rotations included in the fit (in the latter case, an isosceles triangle of $20^{\circ}$ half-angle was fitted in all cases) are also shown in figure 7 . The errors without rotations included grow almost as quickly as the corresponding edge-tracking curve, but by including rotations, the error can be reduced to the order of one thousandth of a pixel, similar to the noise error that is likely to be present under typical conditions. Even with rotations included, the error is still observed to grow slightly with increasing rotation angle: this might be an artifact of the method used to construct the artificial cone images, in particular, the neglecting of edge curvature within each pixel on the cone base. The accuracy in determining the outof-plane rotational angle in these measurements was slightly lower than in-plane rotational measurements, as might be expected, but still typically better than $0.0025^{\circ}$.

These results make it clear that caution must be used in applying the present techniques in unmodified form to certain types of edges if the change in outof-plane rotational angle between images is expected to exceed a few hundredths of a degree. Edges on flat

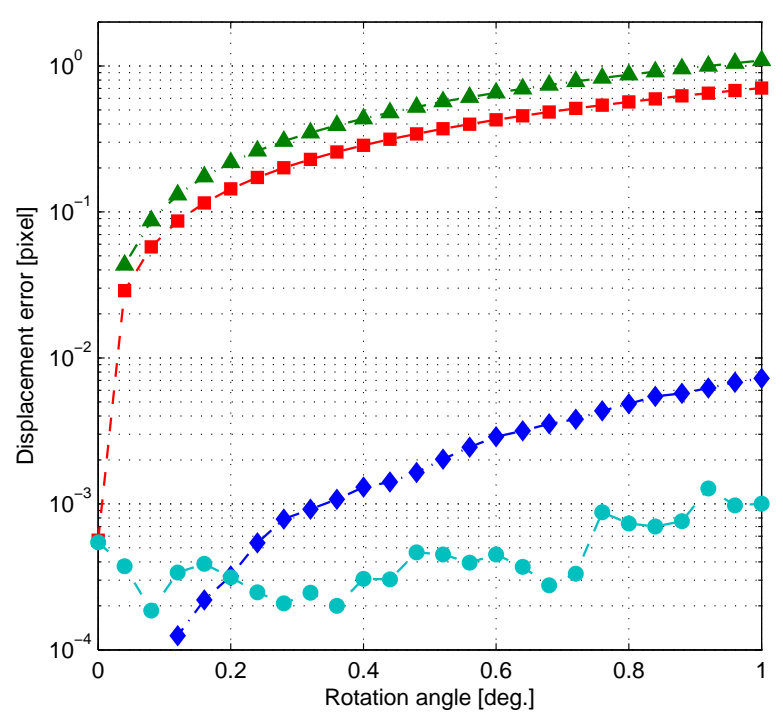

Fig. 7 Displacement errors for a noise-free $20^{\circ}$ half-angle, 100 pixel base radius cone undergoing out-of-plane rotations, using several variants of tracking techniques: $\diamond$, edge-tracking, base edge points included; $\triangle$, edge-tracking, base edge points excluded; $\square$, analytic-fitting with no out-of-plane rotations; $\circ$, analytic-fitting including out-of-plane rotations.

surfaces normal to the imaging plane should be treated particularly carefully.

The cone results may also be used to estimate the parallax errors that would result from using such a geometry with a backlighting arrangement (see the discussion in section 3.2). If, for example, a cone of $50 \mathrm{~mm}$ base diameter located $50 \mathrm{~cm}$ from the imaging device were moved from a position in which its vertex lay on the centerline of the imaging device to one in which its base lay on the centerline, the change in the apparent angle of the base section will be approximately $0.4^{\circ}$. From figure 7 , this will produce an error in the position determination of 0.43 and 0.29 pixels for edge-tracking and analytic-fitting, respectively. As the distance traveled here will correspond to 275 pixels, the errors in the measured displacements will be approximately $0.16 \%$ and $0.1 \%$ respectively, which should be small enough for most applications. A negligible error would result if edge-tracking were applied only to the straight edges. These errors can be expected to scale roughly linearly with the ratio of the cone size to the distance to the imaging device.

\subsection{Experiments with a fixed sphere}

To verify that the artificial image analysis was providing a realistic assessment of the accuracies of the various tracking techniques, calibration measurements were 
carried out with a physical sphere. The $40 \mathrm{~mm}$-diameter precision-machined sphere was attached to a motorized linear displacement stage, with a minimum displacement increment of $1 \mu \mathrm{m}$, and visualized by means of the backlighting arrangement shown in figure 8 . The digital camera used in these experiments (and in all experiments described hereinafter) was a Shimadzu HPV1. The resolution of this camera is $312 \times 260$ pixels and it is capable of capturing 102 images at frame rates of up to $1 \mathrm{MHz}$, though much lower rates were used in the present work. In these sphere experiments, a telephoto lens was attached to the camera, resulting in a resolution of approximately $0.3 \mathrm{~mm} /$ pixel (giving a sphere radius in image dimensions of 67.7 pixels.)

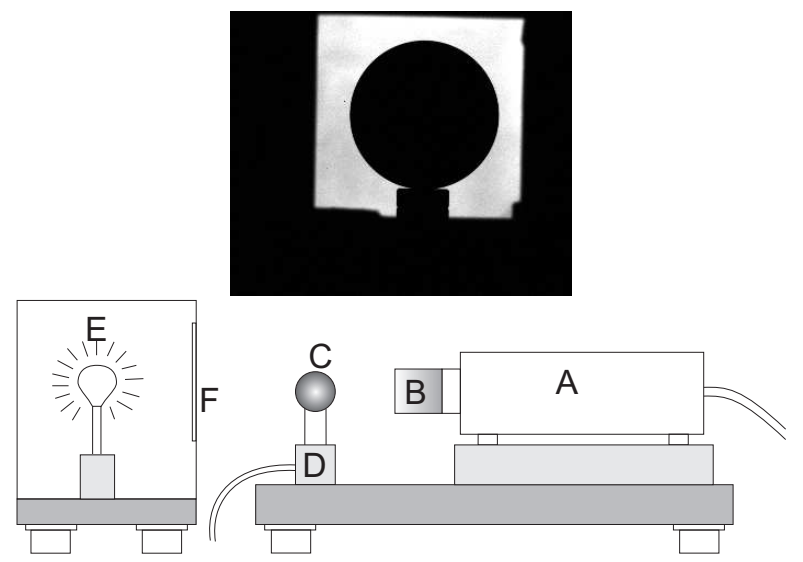

Fig. 8 Sample image and apparatus for sphere experiments: (A) Shimadzu HPV-1 high-speed digital camera; (B) zoom lens; (C) precision-machined $40 \mathrm{~mm}$-diameter sphere; (D) motorized linear displacement stage; (E) light source; (F) Spectralon lightdiffusing material.

Results from one series of experiments, in which the sphere was moved in $5 \mu \mathrm{m}$ increments over a range of $350 \mu \mathrm{m}$, is shown in figure 9. The error in the displacement determination for each of the three applicable techniques is plotted against the inputted displacement. At each displacement value, the mean and standard deviation over 50 images were calculated. In the top curve, obtained from raw images using analytic-fitting, a wave-like profile of amplitude $\sim 1.5 \mu \mathrm{m}$ and with a period of approximately one pixel is apparent. This trend was determined to result from the nonlinear response of the camera CCD, and a calibration using an Ulbricht's sphere was thus carried out. Results for the three techniques using the calibrated images are shown in the lower three curves. The wave-like trend is seen to become much less pronounced, with the points lying closer to the respective zero than previously; the RMS deviation of the mean values for all three calibrated profiles is now $0.45 \mu \mathrm{m}$, compared to $0.80 \mu \mathrm{m}$ for the uncali- brated profile. The calibration has slightly increased the average standard deviation, however, from 0.6 to $0.85 \mu \mathrm{m}$. The corresponding values for the edge-tracking and spline-fitting techniques are 1.02 and $1.05 \mu \mathrm{m}$, respectively. The slightly improved precision of analyticfitting in comparison to the other techniques is consistent with the findings of the preceding artificial image analysis. Further, a standard deviation of $1 \mu \mathrm{m}$ here corresponds to an accuracy of 3 thousandths of a pixel, suggesting that the artificial image analysis gives a realistic indication of the realizable accuracy (the image noise in these measurements, while difficult to give a precise value for, was of the order of $2 \%$ ).

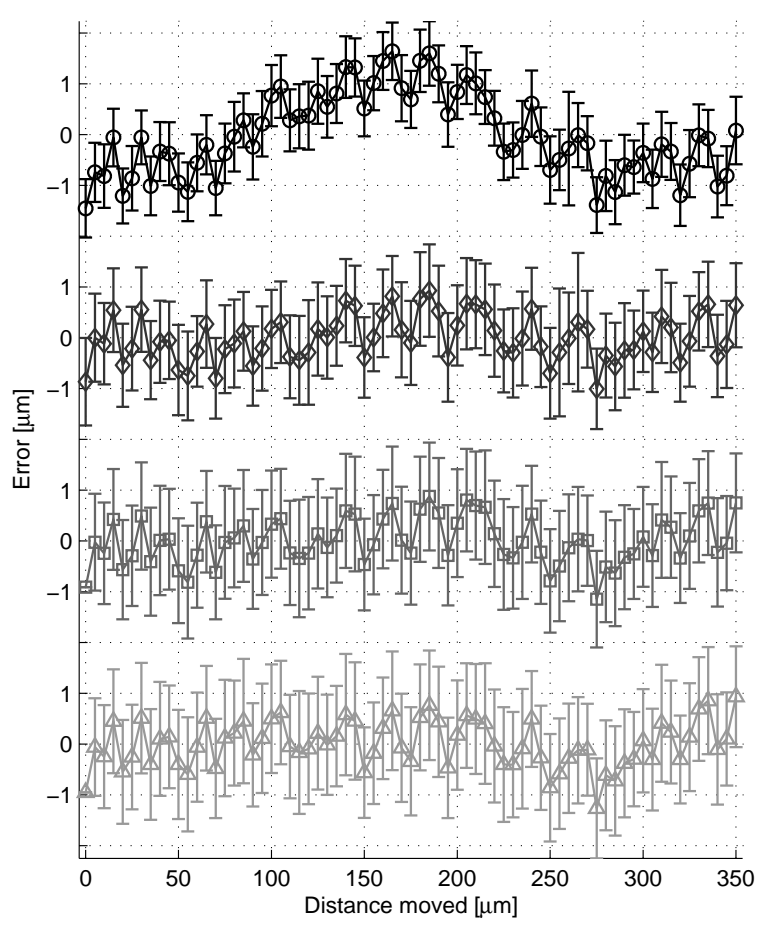

Fig. 9 Errors in displacement measurements of the fixed sphere with a linear stage. From top to bottom: analytic-fitting (uncalibrated for CCD nonlinearity), analytic-fitting (calibrated), edgetracking (calibrated), spline-fitting (calibrated). The zero in each case is set to to the mean error value.

\subsection{Effect of camera pixel structure}

Each of the least-squares fitting techniques described in this article relies on an accurate subpixel-resolution detection of the edge locations, and it has been assumed in the foregoing text that the imaging device and edge-detection software are capable of providing 
this. In this context, it is relevant to note that the method employed to construct the artificial images in section 4.1 implicitly assumed a full-frame device architecture. However, typical high-speed devices have a pixel geometry in which the photosensitive area of the pixel is somewhat below $100 \%$. The in-situ storage image sensor (IS-CCD) in the Shimadzu camera employed in this work is a rather extreme example of this, with a fill factor of only approximately $13 \%$ in the horizontal direction (Etoh et al, 2003). Thus, particularly for sharply-defined vertical edges, one might expect that effective subpixel resolution is not achievable, as movement of the edge within the non-photosensitive area of the pixel will not result in any change in the intensity reading.

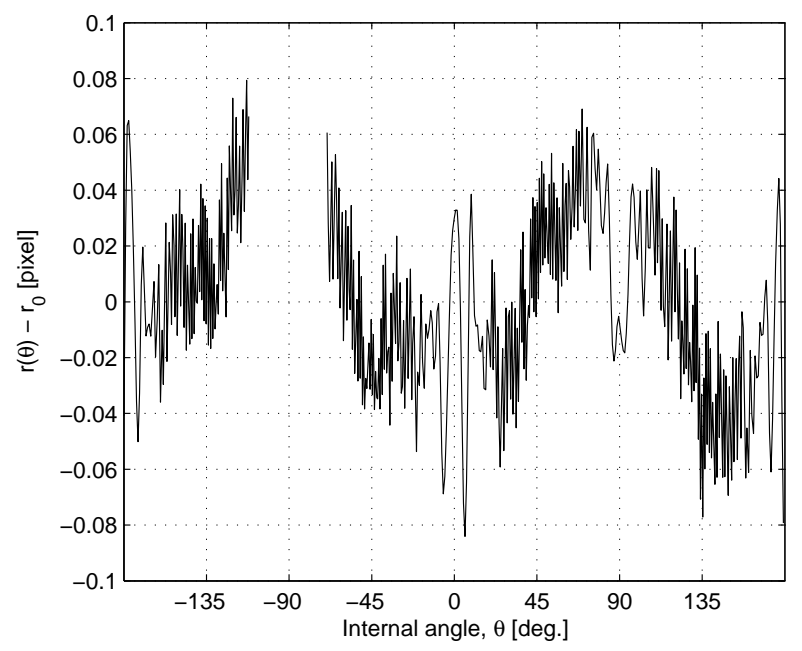

Fig. 10 Deviation of the detected edge radius from the fitted mean radius for a typical image from the calibrated sphere measurements in section 4.2 .

In practice, however, this effect does not seem to be so problematic. In figure 10 is shown the deviation in the measured radius of all detected edge points from the overall fitted radius for one of the images in the fixed sphere experiments. Immediately apparent is a slight distortion to the spherical profile resulting from the offcenter position of the sphere; in addition to noise, there are also some systematic deviations close to 0,90 , and $180^{\circ}$, i.e., edge angles that are close to being aligned to the CCD pixel structure. However, these deviations are limited to a maximum of \pm 0.08 pixels, instead of $\sim 0.5$ pixels that would be expected if only pixel resolution detection were being achieved. The reason for this is most likely a slight defocussing of the image edges (together with unavoidable diffraction effects), meaning that the photosensitive area of the pixel experiences a continuous rather than a step change in intensity as the edge passes over it. Such defocussing can be recommended for the present techniques in order to achieve subpixel resolution for non-full-frame imaging devices. If the device architecture is known, it may also be possible to use artificial simulations to construct a look-up table in order to correct for errors in detected edge locations due to the pixel structure; however, in most cases this is unlikely to be necessary.

\section{Applications of tracking techniques}

To demonstrate the capabilities of the newly introduced tracking techniques under real experimental conditions, two applications are described in this section. Both involve experiments in the HEG facility (High Enthalpy shock tunnel, Göttingen) of the German Aerospace Center (Hannemann, 2003). This is a challenging measurement environment because of both the short test time (a few milliseconds) and the luminosity that often accompanies the high temperature gas behind shock waves. In the first application, the model profile is known (and analytically expressible) but the visualized outline incomplete, whereas in the second the model profile is completely unknown.

\subsection{Measurements on a fixed capsule model}

Under aerodynamic loading, even a sting-mounted model will undergo some displacement as the model and supporting structure respond to the applied forces. In the HEG, depending on the flow conditions and the model characteristics, this displacement can be as small as a few tens of microns; resolving it is thus a good test for the present techniques. High-speed visualizations were captured during a series of experiments on a fixed $18 \mathrm{~cm}$-diameter re-entry capsule model, a schematic of which is shown in figure 11. Images from two experiments are also shown: in the first, the Mach number is $M=7.0$, and the reservoir pressure and temperature are $p_{0}=6 \mathrm{MPa}$ and $T_{0}=3900 \mathrm{~K}$, respectively; in the second $M=6.5, p_{0}=85 \mathrm{MPa}$, and $T_{0}=6000 \mathrm{~K}$. For the first condition the entire model outline is visible, but for the second the increased pressure and temperature lead to higher levels of test-gas luminosity, and the front model face is completely obscured. Edge- and corner-tracking were thus employed, and applied only to the rear model edges.

Any two-dimensional rigid-body motion can be decomposed into a rotation about a given origin and a linear translation. Without knowledge of the centerof-rotation, however, determining the individual rotational and translational contributions to the overall mo- 


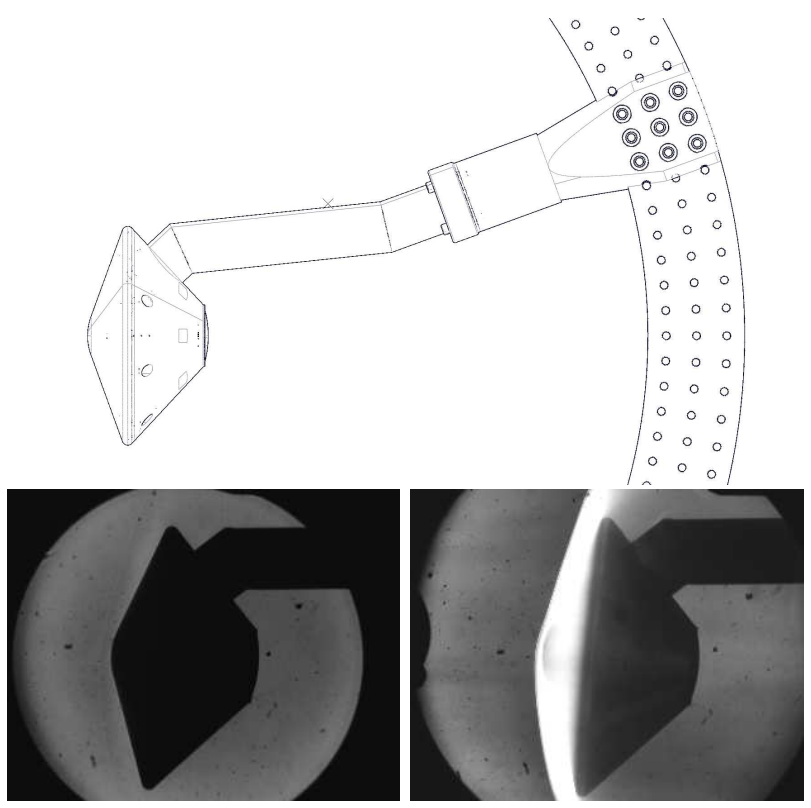

Fig. 11 Schematic and experimental images (at low- and highpressure conditions) of a sting-mounted capsule model in the HEG. The cross just above the sting in the schematic drawing indicates the center-of-rotation that was calculated to result in minimum translational displacements for the low-pressure condition. The magnification in the images is $0.85 \mathrm{~mm} / \mathrm{pixel}$, and they were recorded at $16 \mathrm{kfps}$.

tion is an ill-posed problem. In the present case, if we make the unlikely assumption that the origin lies at the center-of-geometry of the model, effectively minimizing the rotational contribution to the measured displacements, translational components such as those shown in the solid lines of the upper two plots of figure 12 result. These curves are for the low-pressure condition shown in figure 11, and the corresponding rotational motion (which has been smoothed using a moving-average filter with a windowsize of 9 points) is shown in the lowermost plot. The model motion, though only of a few tens of microns, is easily resolved by the tracking techniques. However, for this choice of origin we see that there is a strong correlation between the rotational and translational profiles, suggesting that the rotational contribution to the displacement has not been well captured by this choice.

In fact, the present arrangement, with the forces concentrated on a model at the end of a long sting, is similar to a point load applied to the end of a cantilevered beam; thus, we might expect the observed displacements to be primarily rotational rather than translational in nature, with the effective center-of-rotation lying somewhere on the sting. The position of the rotational origin resulting in the minimum translational displacements was thus calculated: as the $x$-displacement depends almost solely on the $y$-origin location, and vice
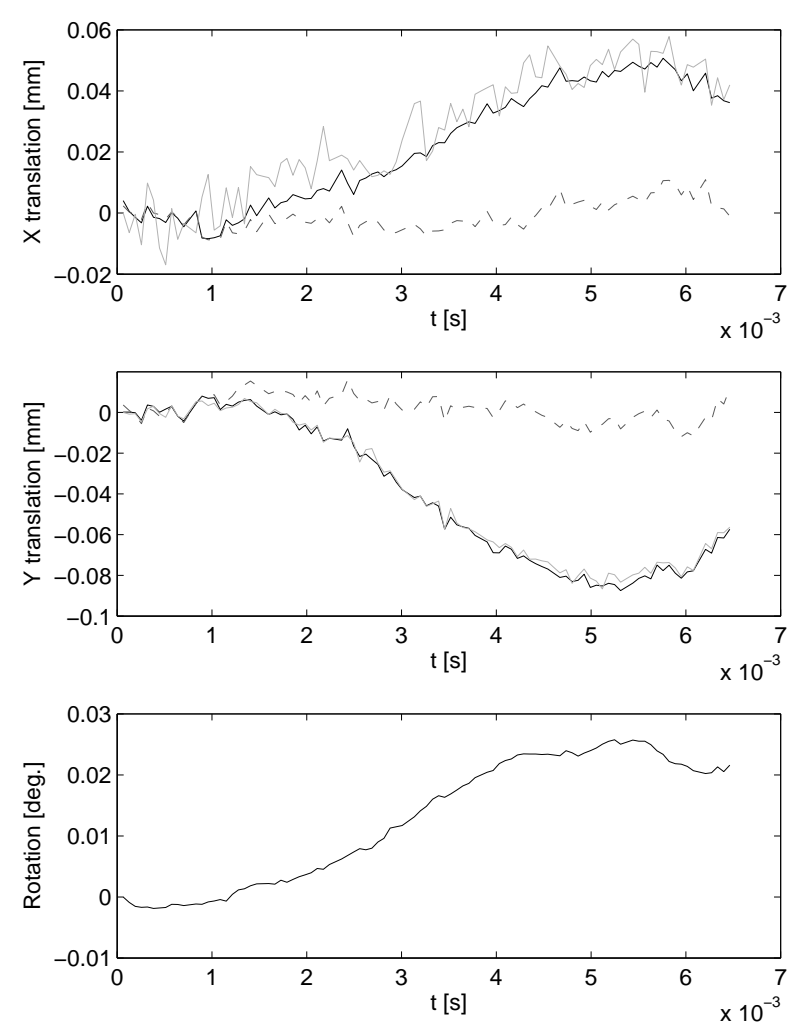

Fig. 12 Measured capsule displacements ( $x$ and $y$ translational, and rotational) for the low-pressure condition shown in figure 11. The solid lines in each of the upper two plots show translations measured by edge-tracking with straight-edge linearization (dark) and corner-tracking (light), assuming the rotational origin to lie at the model center-of-geometry; the dashed lines show these with the origin indicated in figure 11 (edge-tracking only).

versa, this is a simple matter with graphical methods (the linear nature of the two tracking techniques also facilitates repeated calculations). The position thus calculated with edge-tracking is indicated in the schematic of figure 11, and, as expected, lies close to the midpoint of the sting; a similar calculation with corner-tracking yielded a location $23 \mathrm{~mm}$ away. The corresponding $x$ and $y$ translational displacements are plotted in the dashed lines in figure 12 and are seen to be essentially negligible. The assumption that the measured motion results primarily from a rotational deflection of the model on the sting is thus plausible, and these measurements show the versatility of the edge-tracking technique to good effect.

Comparing the edge-tracking and corner-tracking results in figure 12, the $y$-displacements agree closely, but for the $x$-displacements the corner-tracking profile is significantly noisier. The variation in the initial parts of the profiles, before flow arrival, indicate a mea- 
surement uncertainty of $3.7 \mu \mathrm{m}\left(4.4 \times 10^{-3}\right.$ pixels $)$ for edge-tracking, compared to $8.3 \mu \mathrm{m}\left(9.8 \times 10^{-3}\right.$ pixels $)$ for corner-tracking. These observations are consistent with the results of section 4.1.2, and suggests edgetracking to be the preferable alternative of the two.

The image sequence from which the right image in figure 11 came was also analyzed using edge-tracking. The displacements assuming the center-of-mass to coincide with the capsule center-of-geometry were similar to the corresponding profiles in figure 12, but approximately an order of magnitude larger (due to the higher pressure). However, these displacements could not be reduced to a purely rotational motion to the same extent as was possible for the low-pressure condition, suggesting that the response of the model and sting becomes more complex as the aerodynamic loading increases (at the high-pressure condition, the loading on the capsule was estimated from the measured Pitot pressure, assuming a modified Newtonian pressure distribution on the forebody, as $\sim 2200 \mathrm{~N}$ ).

One question regarding the application of the present techniques in compressible flow facilities is the effect that the large density gradients encountered, especially at shocks, will have on the light path and thus the detected edge location. The capsule model in the present measurements, for example, is enveloped by a bow shock extending well downstream of the rear of the body. For measurements within a quasi-steady test time, the shock position, and thus any changes in the detected edge locations should be relatively fixed, meaning that displacement measurements (and derived velocities and accelerations) will be little affected. If the displacement is to be measured relative to an initial flow-off position, however, the influence could be potentially more significant. Such an effect would be expected to manifest itself in a rather abrupt shift in the determined position on arrival of the flow; however, such a shift was in general not observed, suggesting this effect not to be overly problematic in the present experiments. This may be because only the rear edges were used, and in this region downstream the bow shock has already been weakened by the expansion from the capsule shoulder. More care may have to be taken if shocks were to lie closer to the edges employed.

\subsection{Free-flying shuttle model}

As another test case, a $7 \mathrm{~cm}, 30 \mathrm{~g}$ toy space shuttle was mounted in the HEG test section during a tunnel calibration experiment. The model was initially suspended in two places by human hairs, and it was hoped that these would break close to the shuttle body on the arrival of the flow, allowing free flight during the flow time. As may be seen in the images of figure 13, however, some lengths of hair remained attached, and the unsteady forces produced by the flapping of these in the free-stream may have had a non-negligible influence on the model motion.

The movement of the shuttle was measured using both edge-tracking with straight-edge linearization and spline-fitting. For the former, as the overall extent of the motion was of the order of tens of pixels, displacements between consecutive image pairs were calculated, rather than using a single reference image. The noise in the measurements was decreased at the expense of temporal resolution by considering displacements between image pairs separated by up to 4 time-steps.
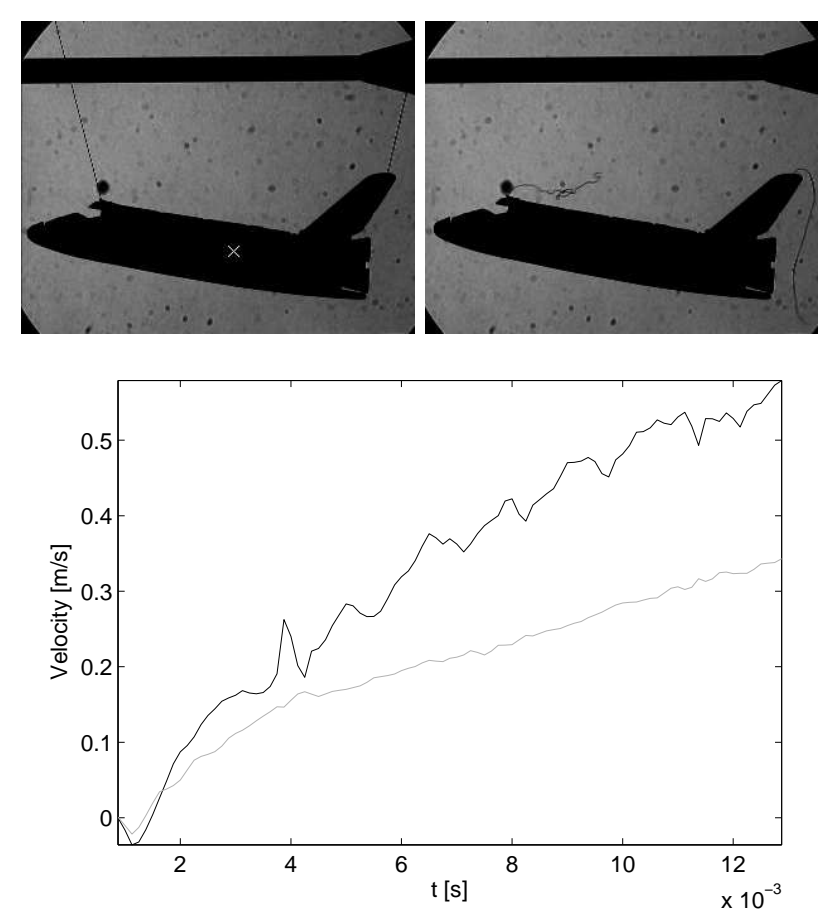

Fig. 13 Images of the shuttle model in the HEG before flow arrival (left) and during the test time (right); and plot of the measured model velocities: (dark) drag; (light) lift. The white cross in the left image indicates the measured center-of-mass. The image frame-rate is $8 \mathrm{kfps}$ and the magnification is $0.255 \mathrm{~mm} / \mathrm{pixel}$. The optical flaws visible in the images (and also in figure 11) are caused by damage to the optical windows.

The shuttle center-of-mass position was determined by hanging the model twice from single threads attached at different locations on the body and capturing images using the setup shown in figure 8 . The centerof-mass was then taken as the point of intersection between the two lines defined by the taut threads, and this is indicated by the cross in the left image of figure 13 .

The drag and lift velocities determined using the edge-tracking technique are shown in figure 13. The lift profile is seen to be less noisy than the drag profile, 
despite the smaller extent of motion: this is because the proportion of the model outline having an edge direction more closely normal to the lift direction is larger than that having an edge direction closely normal to the drag direction. Accelerations, from which the forces can also be easily deduced, were obtained by fitting straight lines to the velocity profile over the time period of interest. For example, over the period 4.4 to $6.0 \mathrm{~ms}$, corresponding approximately to the steady test time, the drag and lift accelerations were $45.8 \pm 8.2$ and $22.6 \pm 0.9 \mathrm{~m} / \mathrm{s}^{2}$, respectively, giving an $\mathrm{L} / \mathrm{D}$ ratio of 0.49 . The variation in the measured pitot pressure during the test period was approximately $3 \%$.

The precision obtained in the lift acceleration measurement $(4 \%)$ is especially noteworthy: while higher precision was obtained over similar time-scales in Laurence and Karl (2010), the accelerations in the present case are approximately two orders of magnitude smaller. Indeed, the characteristic acceleration distance, $a \Delta t^{2} / 2$, is only $29 \mu \mathrm{m}$, which, in context, is smaller than the estimated precision $(50 \mu \mathrm{m})$ of earlier film-based tracking techniques (Canning et al, 1970) or current markerbased techniques (approximately $40 \mu \mathrm{m}$ - see, e.g., Mettler (2010)). Effective displacement errors can be estimated from the residuals to the linear fits of the velocity profiles during the test time: values of $2.0 \mu \mathrm{m}\left(7.7 \times 10^{-3}\right.$ pixels $)$ and $0.3 \mu \mathrm{m}\left(1.1 \times 10^{-3}\right.$ pixels $)$ are obtained for the drag and lift displacements, respectively. These results give some further confirmation that it is possible, under experimental conditions, to realize the capabilities of the techniques suggested by the idealized analysis earlier in this article.

Displacements determined from both edge-tracking and spline-fitting are shown in figure 14. The lift profiles are generally in good agreement, but the drag profiles diverge noticeably from around $9 \mathrm{~ms}$. The spline-fitting measurements are significantly noisier, and the iterative fitting routine would not converge from $10.7 \mathrm{~ms}$. On comparing the edge points detected at different times in the image sequence, it was found that the model profile was changing, consistent with a rolling motion of the shuttle. This was most noticeable in later images, and is likely to be the reason for the observed discrepancy, as well as the failure of the fitting routine to converge. The results of neither technique can thus be considered valid at these later times.

\section{Discussion}

To conclude, I briefly discuss the relative merits and deficiencies of the four tracking techniques investigated in this work.
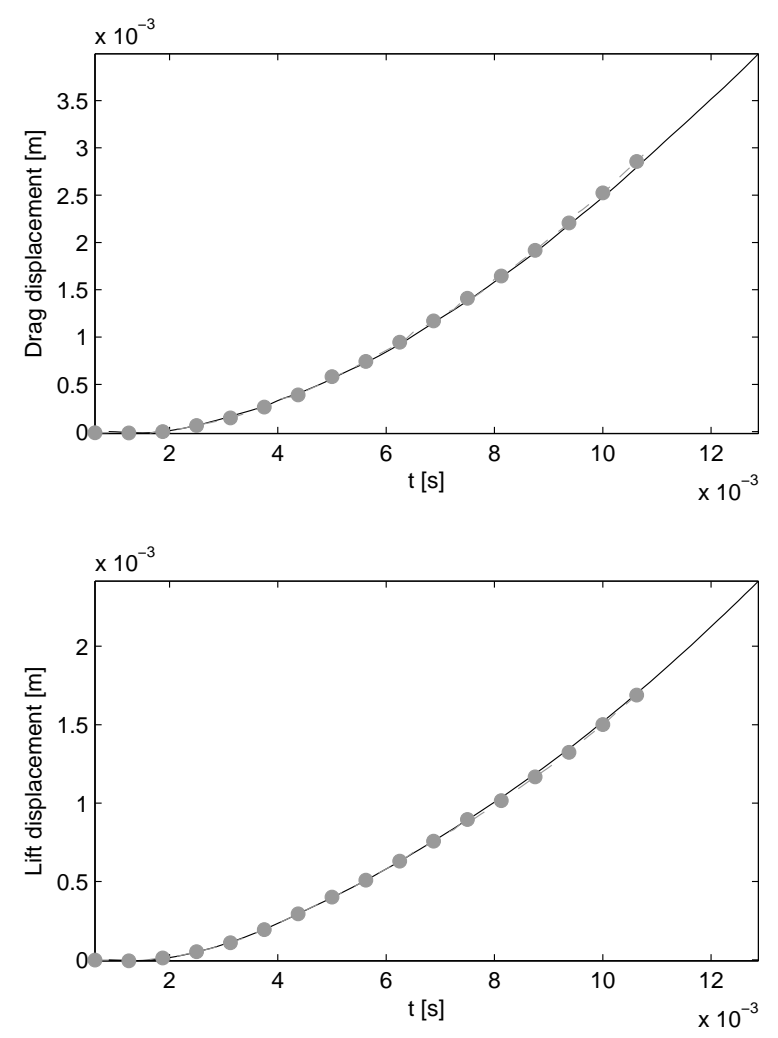

Fig. 14 Measured displacements of the shuttle center-of-mass calculated using edge-tracking (dark, solid) and spline-fitting (light, dashed-circle).

In situations in which the analytic-fitting technique can be applied (i.e., model cross section expressible analytically; relatively complete profile visible), it appears to be the best option for obtaining precise measurements. First, absolute position measurements are obtained, which may be particularly useful if the relative positions of multiple objects are to be determined. Furthermore, if the model is freely moving and the physical center-of-mass is known, the center-of-rotation in image coordinates is immediately available; the scaling factor between image and physical dimensions can also be calculated automatically as part of the fitting routine (for other methods of determining the scaling factor, see Bernstein (1975), for example). Second, at low image-noise levels, the artificial image analysis here indicates that displacements can be calculated at least as precisely as with the other techniques, though there was also some indication that in certain cases analyticfitting might be more sensitive than edge-tracking to systematic errors in the edge locations. Finally, if measurements were to be extended by the addition of a second imaging device to include the remaining components of motion, this technique is presumably the most 
suitable. The major disadvantage of the analytic-fitting technique (other than the factors that might preclude its use) is the need for a time-consuming iterative fitting procedure for all but the simplest of geometries.

Edge-tracking appears to be a useful alternative to analytic-fitting (more so than the other techniques), and has a wider range of applicability. It is relatively easy to implement, versatile in terms of the edge sections that may be used, and was found to be at least as accurate as spline-fitting and corner-tracking. A further advantage over spline-fitting (and also analytic-fitting) is that the fitting procedure can typically be reduced to solving a sequence of linear least-squares problems, with the benefits in terms of computational cost that this brings.

The spline-fitting technique is less useful than either of the preceding techniques for the types of measurements described in this article, as it combines most of their disadvantages with few of the advantages. In particular, the fitting procedure is iterative, and thus computationally intensive, regardless of the model shape; also, unlike the edge-tracking technique, a relatively complete visualized profile is necessary for convergence. Spline-fitting is probably most useful as a check on the results obtained with edge-tracking.

Finally, the corner-tracking technique is applicable only to a certain class of geometries. In its favor are simplicity of implementation and the fact that it is always linear, but its accuracy appears limited for bodies of small image dimensions, and it is relatively sensitive to image noise.

Acknowledgements The author wishes to acknowledge the assistance of Jan Martinez Schramm in carrying out the experiments in section 5; and to thank Sabrina Rutschmann for performing the fixed sphere experiments and the intensity calibration, and for contributing her hair to the shuttle experiment. Useful discussions were also had with Jeremy Wolfram.

\section{References}

Ballard D (1981) Generalizing the Hough transform to detect arbitrary shapes. Pattern Recognition 12(2):111-122

Bernstein L (1975) Force measurements in shortduration hypersonic facilities. AGARDograph No 214

Canning T, Seiff A, James C (1970) Ballistic-range technology. AGARDograph No 138

Canny J (1986) A computational approach to edge detection. IEEE Trans Pattern Anal Machine Intell 8(6):679-698

de Castro E, Morandi C (1987) Registration of translated and rotated images using finite Fourier trans- forms. IEEE Trans Pattern Anal Machine Intell PAMI-9(5):700-703

Etoh T, Poggemann D, Kreider G, Mutoh H, Theuwissen A, Ruccckelshausen A, Kondo Y, Maruno H, Tabuko K, Soya H, Tajehara K, Okinaka T, Takano Y (2003) An image sensor which captures 100 consecutive frames at 1,000,000 frames/s. IEEE Trans Electron Dev 50(1):144-151

Gander W, Golub G, Strebel R (1994) Least-squares fitting of circles and ellipses. BIT 34:558-578

Hannemann K (2003) High enthalpy flows in the HEG shock tunnel: Experiment and numerical rebuilding. In: 41st AIAA Aerospace Sciences Meeting and Exhibit, Reno, Nevada

Laurence S, Hornung H (2009) Image-based force and moment measurement in hypersonic facilities. Exp Fluids 46:343-353

Laurence S, Karl S (2010) An improved visualizationbased force-measurement technique for shortduration hypersonic facilities. Exp Fluids 48:949-965

Marston J, Yong W, Thoroddsen S (2010) Direct verification of the lubrication force on a sphere travelling through a viscous film upon approach to a solid wall. J Fluid Mech 655:515-526

Mettler B (2010) Extracting micro air vehicles aerodynamic forces and coefficients in free flight using visual motion tracking techniques. Exp Fluids 49:557-569

Settles G (2006) Schlieren and shadowgraph techniques. Springer, NY

Spoor C, Veldpaus F (1980) Rigid body motion calculated from spatial co-ordinates of markers. J Biomechanics 13:391-393

Thoroddsen S, Etoh T, Takehara K (2008) High-speed imaging of drops and bubbles. Ann Rev Fluid Mech 40:257-285

Verbeek P, van Vliet L (1994) On the location error of curved edges in low-pass filtered 2-d and 3-d images. IEEE Trans Pattern Anal Machine Intell 16(7):726733

Warren W, Kaegi E, Geiger R (1961) Shock tunnel experimental techniques for force and moment and surface flow direction measurements. ARS J 31(1):82-83

Yang FL, Hunt M (2006) Dynamics of particle-particle collisions in a viscous liquid. Phys Fluids 18(121506)

Zhang Z (1997) Parameter estimation techniques: A tutorial with application to conic fitting. Image Vision Comput 15(1):59-76 